Bull. Mater. Sci., Vol. 17, No. 6, November 1994, pp. 875-881. (C Printed in India.

\title{
Studies on high temperature deformation behaviour of 3Y-TZP ceramics
}

\author{
D D UPADHYAYA, S K ROY, G K DEY and S BANERJEE \\ Metallurgy Division, Bhabha Atomic Research Centre, Bombay 400085 , India
}

\begin{abstract}
The present work reports the results on the deformation behaviour of $\mathrm{ZrO}_{2}-3$ $\mathrm{mol} \% \mathrm{Y}_{2} \mathrm{O}_{3}$ (3Y-TZP) ceramics which were prepared by pressureless sintering at $1400^{\circ} \mathrm{C}$. Dense, cylindrical samples were subjected to uniaxial compression tests under a constant stress of $15 \mathrm{MPa}$ in the temperature range of $1200-1400^{\circ} \mathrm{C}$. The ceramics exhibit considerable ductility, attaining over $60 \%$ true strain without any edge cracking. Microstructural changes due to interaction of grain boundary viscous phase with the ultrafine and equiaxed grains were analyzed by transmission electron microscopy. Results show the grain boundary sliding accompanied by a diffusion accommodation process as the predominant deformation mechanism in these ceramics.
\end{abstract}

Keywords. Yttria stabilized zirconia; superplasticity; microstructure; TEM.

\section{Introduction}

$\mathrm{Y}_{2} \mathrm{O}_{3}$ stabilized tetragonal $\mathrm{ZrO}_{2}$ polycrystals (Y-TZP) form one important class of transformation toughened ceramics. These $\mathrm{ZrO}_{2}$ based alloys are promising structural materials for various high performance applications because of their excellent mechanical properties such as strength and toughness. It has also been observed that these ceramics fulfil many of the microstructural prerequisites that have been established for superplasticity in metals and alloys. Subjecting ceramics to deformation forming is thus an important recent advancement in fabrication technology (Panda et al 1988; Chen and Xue 1990). Dense Y-TZP ceramics obtained on sintering at moderate temperatures $\left(\sim 1400^{\circ} \mathrm{C}\right)$ exhibit a homogeneous single phase (tetragonal$\left.\mathrm{ZrO}_{2}\right)$, microstructure consisting of ultrafine $(<1.0 \mu \mathrm{m})$ and equiaxed grains. The impurities introduced during processing often contribute to the grain boundary liquid phase by the formation of low temperature eutectic melts (Lang et al 1986). Extensive studies have been carried out in tailoring a microstructure which is stable against coarsening during deformation at elevated temperatures. A suitable modification of grain boundary chemistry by incorporating some system specific dopants $(\mathrm{MnO}, \mathrm{CoO})$ and by providing a composite configuration with other oxides (e.g. $\mathrm{Al}_{2} \mathrm{O}_{3}$ ) is usually followed in these systems (Kimura et al 1988; Wakai 1989; French et al 1990; Upadhyaya et al 1993a, b).

The mechanism of superplasticity in ceramics is not fully understood. It is generally explained in terms of models based on the grain boundary sliding or diffusional flow mechanisms (Wang and Raj 1984; Wakai et al 1989). The constitutive equation for deformation in polycrystalline samples at elevated temperature has the form:

$$
\varepsilon^{\cdot}=A\left(\sigma^{n} / d^{n}\right) \exp (-Q / R T)
$$


where $A$ is the deformation mechanism dependent function, $d$ the mean grain size, $p$ the grain size exponent and $n$ the stress exponent (reciprocal of strain rate sensitivity). The other symbols in above equation have the usual significance.

For Y-TZP ceramics, a large discrepancy appears in the value of $n$ reported by different authors (from 1.1 to 3.5 ) (Wakai 1989). The difference in behaviour and a predominantly non-Newtonian flow $(n>1)$ mechanism is likely to be dependent on the concentration and nature of the grain boundary glassy phase. Present ' work tries to analyze the interaction mechanism of the two microstructural parameters, viz. the rigid grains and soft boundary phase (glassy) in 3Y-TZP ceramics at high temperatures, under compressive loading conditions using TEM as a tool.

\section{Experimental}

The wet chemistry, co-precipitation route was employed to prepare fine powder of $\mathrm{ZrO}_{2}-3 \mathrm{~mol} \% \mathrm{Y}_{2} \mathrm{O}_{3}$. The starting material was $3 \mathrm{~N}$ purity salts of $\mathrm{ZrOCl}_{2} \cdot 8 \mathrm{H}_{2} \mathrm{O}$ and $\mathrm{Y}(\mathrm{NO})_{3} \cdot 5 \mathrm{H}_{2} \mathrm{O}$. Mixed solutions $(0.5 \mathrm{M})$ were added drop wise into a vigorously stirred liquor ammonia bath which produced a white, gelatinous substance precipitated. These hydroxide gels were filtered, washed, dehydrated with ethanol and dried in air oven at $110^{\circ} \mathrm{C}$. The dried mass was pulverized, calcined at $600^{\circ} \mathrm{C}$ for $3 \mathrm{~h}$ and ball milled for $8 \mathrm{~h}$ using Y-TZP grinding medium.

Cold compacted (84 MPa) samples on sintering at $1400^{\circ} \mathrm{C}$ for $2 \mathrm{~h}$ attained nearly full (98\% TD) densification. Test samples (10 $\mathrm{mm}$ height $\times 10 \mathrm{~mm}$ diameter) were prepared with their parallel faces fine polished and coated with BN powder. The uniaxial compression experiments were carried out between 1200 and $1400^{\circ} \mathrm{C}$ in vacuum $\left(10^{-3} \mathrm{~Pa}\right)$ at a constant load of $15 \mathrm{MPa}$. Tests were terminated at a true strain of about -0.6 in order to avoid nonuniform stresses and friction conditions. For microstructural investigations the deformed samples were diamond sawed to about $150 \mu \mathrm{m}$ sections. The $3 \mathrm{~mm}$ diameter discs were subsequently prepared by ultrasonic cutter and further mechanically thinned using disc grinder followed by dimple grinding using diamond paste. The final thinning to electron transparency was carried out in an argon ion mill operating at $4 \mathrm{kV}$. Carbon was deposited on the foils to prevent static charge accumulation during observation. A JEOL 2000 FX microscope was used for studying the microstructure.

\section{Results and discussion}

\subsection{Undeformed $3 Y-T Z P$ ceramics}

Microstructural features of the reference material are as shown by the typical micrograph in figure 1 . It essentially shows a very fine grain structure $(-250 \mathrm{~nm})$ having a narrow size distribution. The as-sintered samples showed a dense structure, such that almost no porosity is found in both inter and transgranular regions. The rounded shape of the grains is characteristic of the milled Y-TZP powders which have undergone densification by liquid phase sintering mode (Lang et al 1986; Lin et al 1990). The amorphous phase traps impurities and mainly segregates at the grain trijunction and also forming an apparently continuous intergranular thin film.

Presence of glassy phase is beneficial in relieving the residual stresses developed 


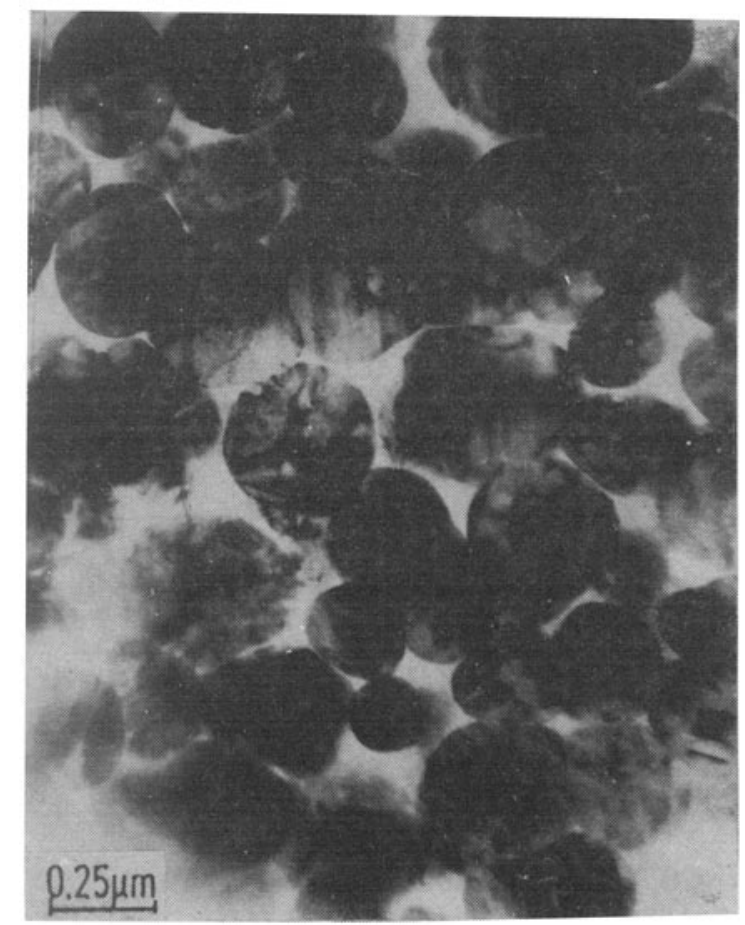

Figure 1. Transmission electron micrograph of undeformed $3 Y-T Z P$ showing the rounded grains and glassy grain boundary phase.

during cooling due to anisotropic thermal contraction of $t-\mathrm{ZrO}_{2}$ grains (Lin et al 1990). In the present material, the grains were thus free from tweed like structure due to strain field contrast found in high purity Y-TZP ceramics having straight boundaries with sharp apexes (Amana et al 1992). The grains rather exhibited a distribution of three twin related variants of $t-\mathrm{ZrO}_{2}$. The frequency of occurrence of twins was very large with most of the twins revealing the presence of more than one variant in each grain with the different variants sometimes lying in a triangular arrangement.

Another significant observation was with respect to the occasional presence (in about $5 \%$ of the specimen area scanned) of the large $(>1 \mu \mathrm{m})$ grains surrounded by an ensemble of significantly smaller size grains (figure 2). This anomalous growth of solute rich $c-\mathrm{ZrO}_{2}$ grains is generally observed in Y-TZP on high temperature annealing $\left(1500^{\circ} \mathrm{C}\right)$. Development of such microduplex structure due to equilibrium partitioning of yttria was found to be very effective in maintaining the fine grain size of Y-TZP ceramics (Lang et al 1985; Lang 1986). The large grains of the $c-\mathrm{ZrO}_{2}$ were irregularly shaped unlike the smaller grains of $t-\mathrm{ZrO}_{2}$ which were mostly spherical. Twin like features could be seen in some of the $c-\mathrm{ZrO}_{2}$ grains.

\subsection{Microstructural evolution after deformation}

Dense 3Y-TZP samples in the shape of right cylinders were compression-tested 
uniaxially at $1200-1400^{\circ} \mathrm{C}$ which is the thermodynamic stability range of tetragonal phase of $\mathrm{ZrO}_{2}$. The absence of transformation toughening in conjunction with the favourable microstructural parameters viz. ultrafine grain size and residual grain boundary viscous phase imparts appreciable plastic deformation before failure. The change in microstructure is as shown in figure $3(\mathrm{a}-\mathrm{c})$ for the sample strained to $60 \%$ at $1400^{\circ} \mathrm{C}$. It is observed that some amount of coarsening of grains takes place without affecting their isometric shape. The glassy phase is relocated (squeezed) in the form of thin intergranular film. It was possible to see grain boundary trijunctions having very little or no amorphous phase in between.

About the high temperature plastic deformation of bulk $\mathrm{ZrO}_{2}$ alloy single crystals, considerable work has been done over the years. It is mostly the work of Heuer and coworkers (Dominguez-Rodriguez et al 1986; Cheong et al 1989, 1991; Fries et al 1989; Heuer et al 1989) which establishes that in Y-CSZ the deformation behaviour is very sensitive to crystal orientation together with other parameters, e.g. Solute concentration, temperature and strain rate. Significant solid solution strengthening is observed over the range of compositions studied $(9.4-21 \mathrm{~mol} \%$ $\mathrm{Y}_{2} \mathrm{O}_{3}$ ). The yield and flow stress increase with increasing $\mathrm{Y}_{2} \mathrm{O}_{3}$ content. Similarly,

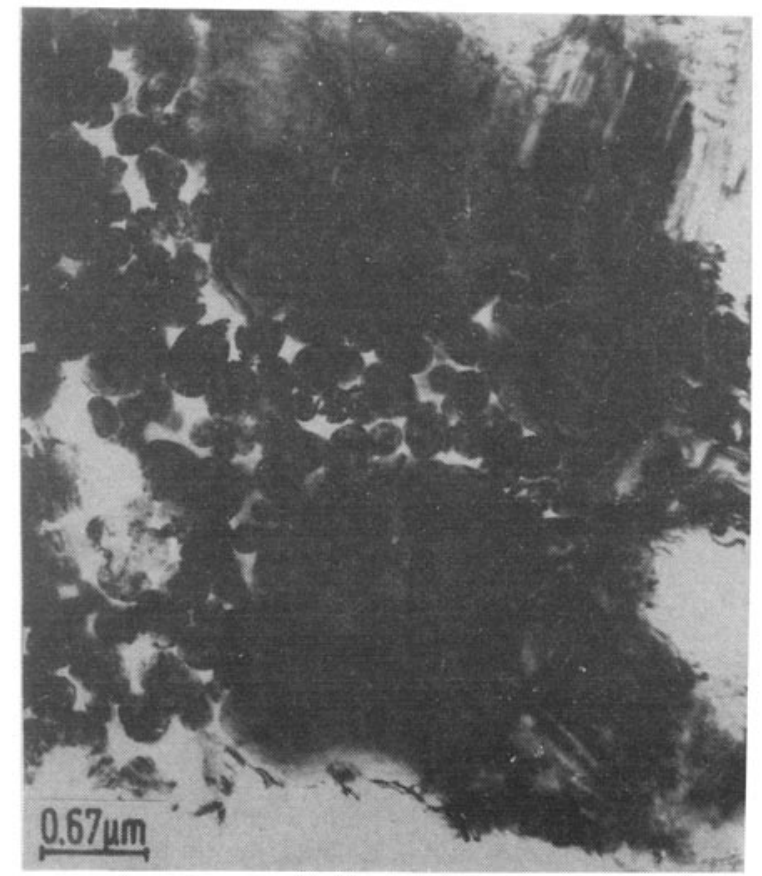

Figure 2. Bright field micrograph showing an occasional bimodal grain size distribution containing coarse $c-\mathrm{ZrO}_{2}$ grain surrounded by a much finer $t-\mathrm{ZrO}_{2}$ grain cluster.

Figure 3. ' Microstructural changes after plastic deformation of $3 Y$.TZP $(\varepsilon=60 \%, T=$ $1400^{\circ} \mathrm{C}, \sigma=15 \mathrm{MPa}$ ). a. Grains are coarsened but the isometric shape is retained, $b$. considerable squeezing of the grain boundary liquid into the residual pores and $c$. stress induced reorientation of ferroelastic domains in $t-\mathrm{ZrO}_{2}$. Two sets of twin variants grow at the expense of third set to facilitate the stress accommodation. 
High temperature deformation of $3 Y-T Z P$ ceramics

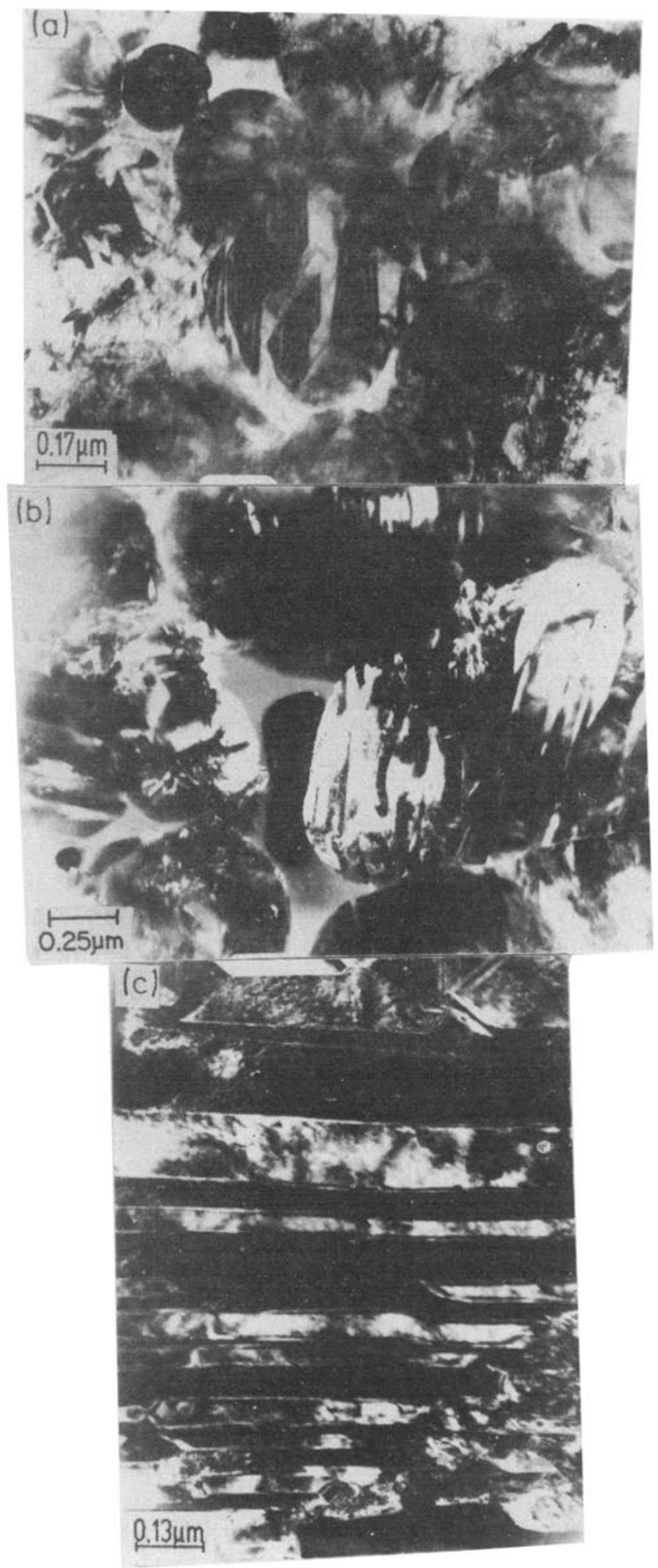


in two-phase Y-PSZ single crystals the phenomenon of precipitation hardening is encountered. In these experiments the stresses employed are generally large $(>200$ $\mathrm{MPa}$ ) and alloy crystals exhibit predominantly a shear band deformation mode accompanied by excessive dislocation activity. Lankford et al (1988) supplemented these results by introducing the contributions from transformation plasticity and ferroelastic domain switching.

In the case of polycrystalline $\mathrm{ZrO}_{2}$ based ceramics the situation is very complex due to grain boundary lubrication by an ubiquitous amorphous phase, for sliding mode to dominate. This permits only a phenomenological explanation by attributing it as the constituent responsible for the viscous-like flow during compression. Maintaining equiaxed grain shape even after extensive deformation is an important aspect which generally suggests a grain intercalation mechanism operating as those visualized in superplastic deformation conditions. An elongation of the grains in the direction perpendicular to the loading axis is predicted if diffusional creep prevails which is contrary to present observations. TEM also revealed that grains were nearly free of dislocations indicating a somewhat minor contribution of dislocation motion in the creep of Y-TZP, in the temperature and stress regimes employed in this work. Though the extent of deformation of the $t-\mathrm{ZrO}_{2}$ grains was extremely small, the boundaries between the $t-\mathrm{ZrO}_{2}$ and $c-\mathrm{ZrO}_{2}$ grains showed considerable amount of waviness in some regions.

The grain boundary amorphous phase has a dual role in influencing the deformation behaviour of Y-TZP. It invariably leads to a strain enhanced dynamic grain growth because of high homologous temperature employed $(\sim 0.5 \mathrm{Tm})$. This suggests that grain boundary sliding is accommodated by interface-reaction controlled diffusional creep in the presence of glassy aluminosilicate phase. The model of Pharr and Ashby (1983) describes the liquid enhanced creep on the basis of repetitive cycles of plasticity and dissolution. The macroscopic yielding of 3Y-TZP thus can be rationalized in terms of micrograin superplasticity which is due to the superposition of various mechanisms viz. grain boundary sliding, grain rotation, diffusional accommodation and viscous flow. In addition to this the possible role of important subgrain features of $t-\mathrm{ZrO}_{2}$, i.e. the ferroelastic twin rotation also needs to be evaluated. The domain switching is an intrinsic, thermally activated process involving migration of the interfaces separating the domains. This migration process generates a shear distortion in the fluorite lattice.

TEM is a useful technique for analyzing the change in twinning mode after deformation. Unlike the $t-\mathrm{ZrO}_{2}$ grains of the undeformed control sample the grains of deformed specimens showed generally a single twin variant (figure $3 \mathrm{c}$ ). This fact could be ascertained from the bright and dark field micrographs and corresponding selected area diffraction patterns. Presence of single twin variant was indicative of the occurrence of domain switching. This twin reversal is the unique stress accommodation parameter for 3Y-TZP ceramics and can contribute significantly towards deformation (Lankford et al 1988).

The foregoing discussions have pointed to the role of multiple parameters involved in the deformation processing of 3Y-TZP ceramics. From the mechanistic standpoint it can be understood to be completed essentially in three steps. In the initial stage, a lubricated flow begins due to liquid film migration and partial dewetting of the grain boundaries. The flow is sustained in the intermediate stage by solutionprecipitation creep. A redistribution of liquid into the residual pores and other 
lower energy sites, softens the stress concentration at the grain interfaces. In the final stage the solid-solid contacts are established between the grains and the above mentioned twin reversal process is activated. The different values of strain rate sensitivity exponent measured by different authors for their materials can be attributed to the difference in critical balance obtained between various stress relieving processes.

\section{Summary}

The present work deals with the superplastic deformation of 3Y-TZP ceramics during compressive creep testing up to true strain near $60 \%$ and the resultant microstructural development of the material. TEM results suggest a three-step process sequence basically comprising the redistribution of the grain boundary amorphous phase, the concurrent grain growth due to deformation enhanced diffusion and a stress accommodation due to subgrain ferroelastic domain switching of $t-\mathrm{ZrO}_{2}$. The grain boundary amorphous phase plays a dominant role in controlling the flow properties of 3Y-TZP. The difference in its chemistry thus explains the discrepancy in the reported value of the deformation parameters.

\section{Acknowledgements}

The authors thank $\operatorname{Dr} \mathrm{R}$ Bhat and $\mathrm{Mr} \mathrm{R} \mathrm{K}$ Fotedar for their assistance in powder synthesis and compression testing.

\section{References}

Amana B, Duclos R and Crompon C 1992 Ceram. Int. 181285

Chen I W and Xue L A 1990 J. Am. Ceram. Soc. 732585

Cheong D-S, Dominguez-Rodrigues A and Heuer A H 1989 Philos. Mag. 60123

Cheong D-S, Dominguez-Rodriguez A and Heuer A H 1991 Philos. Mag. 63377

Dominguez-Rodriguez A, Lanteri $V$ and Heuer A H $1986 \mathrm{~J}$. Am. Ceram. Soc. 69285

French J D, Harmer M P, Chan H M and Miller G A 1990 J. Am. Cerum. Soc. 732508

Fries E, Guiberteau F, Dominguez-Rodriguez A, Cheong D S and Heuer A H 1989 Philos. Mag. 60107

Heuer A H, Lanteri V and Dominguez-Rodriguez A 1989 Acta Metall. 37559

Kimura N, Okamura $\mathrm{H}$ and Morishita J 1988 in Advances in Ceramics; science and technology of zirconia (eds) S Somiya, N Yamamota and H Yanagida (Ohio: Amer. Ceram. Soc.) Vol. 24, p. 183 Lang F F 1986 J. Am. Ceram. Soc. 69240

Lang F F. Marshall D B and Porter J 1985 in Ultrastructure processing of advanced ceramics (eds) J D Mackenzie and D R Ulrich (New York: Wiley) p. 519

Lang F F, Schubert H, Clausen N and Ruhle M 1986 J. Am. Ceram. Soc. 69768

Lankford J, Page R A and Rabenberg L 1988 J. Mater. Sci. 234144

Lin Y J, Angelini P and Mecartney M L $1990 \mathrm{~J}$. Am. Ceram. Soc. 732128

Panda P C, Wang J and Raj R 1988 J. Am. Ceram. Soc. 71 C-507

Pharr G M and Ashby M F 1983 Acta Metall. 31129

Upadhyaya D D, Dalvi P Y and Dey G K 1993a J. Mater. Sci. 286103

Upadhyaya D D, Kutty T R G and Ganguly C 1993b in Science and technology of zirconia- $V$ (eds) S P S Badwal, M J Bannister and R H J Hannink (Basel: Technomic Pub.) p. 310

Wakai F $1989 \mathrm{Br}$. Ceram. Trans. J. 88205

Wakai F, Kodama Y and Nagano T 1989 Jap. J. Appl. Phys. 57

Wang J G and Raj R 1984 J. Am. Ceram. Soc. 67399 